\title{
10-year trends in English primary care glaucoma prescribing
}

\author{
H. D. Jeffry Hogg ${ }^{1,2}$ - Alan Connor ${ }^{2}$
}

Received: 27 June 2019 / Revised: 25 September 2019 / Accepted: 2 October 2019 / Published online: 4 November 2019

(c) The Author(s), under exclusive licence to The Royal College of Ophthalmologists 2019

\begin{abstract}
Background In 2018 NHS prescriptions in England cost $£ 8.83$ billion. Within ophthalmic prescribing, glaucoma is the most costly indication. The 2017 glaucoma NICE guideline shows there is little evidence for clinical preference of particular molecules within a therapeutic class, yet the cost of these products varies greatly. We aim to describe trends in glaucoma prescribing and its relation to recent NICE Guidance.

Methods Prescription cost analyses for England from 2009 to 2018 were reviewed and data concerning items for the treatment of glaucoma were extracted. Costs and prescription frequencies were normalised for inflation and population.

Results The 2018 cost of glaucoma prescribing was $£ 114.2$ million. This cost is $18.1 \%$ lower than in 2009 but the annual number of items prescribed per 10,000 people has increased from 1382 to $1668(20.7 \%)$. This is despite an increased prescription of combination drops from 265 to 478 per 10,000 (80.4\%). Preservative free item prescriptions rose from 1.7\% of total spend in 2009 , at $£ 3.4$ million in 2009 , to $13.9 \%$, in 2018 , at $£ 22.5$ million. Generic items represented $11.7 \%$ of prescriptions in 2009 and $55.2 \%$ in 2018. Around half of glaucoma spending is accounted for by the use of preservative free or branded items in the place of the cheapest item in each therapeutic class.

Conclusions Glaucoma prescribing costs the NHS a great deal. There is a broad trend to generic prescribing as per recent NICE guidance, but significant further costs could be saved with no robustly evidenced clinical consequence.
\end{abstract}

\section{Introduction}

Among Europeans aged 40-80 glaucoma has a prevalence of $2.9 \%$ [1]. Whilst the majority of patients can have their disease halted with topical medication it remains the second leading cause of registration for partial and full sight impairment in the UK [2]. Early identification and treatment of glaucoma and ocular hypertension, a risk factor for the development of glaucoma, is associated with better outcomes. However, it represents a significant financial burden on health services, both from costs related to assessment as well as drug costs.

Supplementary information The online version of this article (https:// doi.org/10.1038/s41433-019-0656-z) contains supplementary material, which is available to authorized users.

H. D. Jeffry Hogg

Jeffry.hogg@ncl.ac.uk

1 The University of Newcastle upon Tyne, Newcastle upon Tyne, Tyne and Wear NE1 7RU, UK

2 The Newcastle upon Tyne Hospitals NHS Foundation Trust, Newcastle upon Tyne, Tyne and Wear NE1 7RU, UK
Between 2000 and 2012 we reported a $67 \%$ increase in glaucoma prescriptions in English primary care [3]. This was in part due to the publication of treatment guidelines by the National Institute for Clinical Excellence (NICE) in 2009 as well as a subsequent change in case finding behaviour by community optometrists. During the same time period there was a $88 \%$ increase in costs with $£ 103.7$ million annual drug cost in 2012 [3]. This increase in drug spending was occurring at a time when pressure was being put on the NHS to restrain spending due to growing financial deficit [4].

We previously reported that medications with patent protection had higher costs than those without and that branded medications were more expensive than their generic counterparts. In recognition of the associated higher cost as well as the fact that there is no obvious therapeutic superiority within each drug class the NICE 2017 guideline recommended that generic medicines should be considered as the first choice treatment [5]. The update also specified that topical treatment escalation should not involve switching a drug for another in the same therapeutic class, but the addition of a drug from a different class. The recommendation for the use of preservative free drops was also broadened to specify patients experiencing 
symptomatic ocular surface disease rather than just those with signs of preservative allergy [5].

This study aims to provide an update of glaucoma prescribing trends along with their associated costs and also to examine how these relate to revised treatment guidelines published by NICE in 2017 [5].

\section{Methods}

Annual prescription cost analysis (PCA) data in the public domain describing primary care prescriptions in England from 2009 to 2018 were accessed [6]. All prescriptions of medications in section 11.6 of the British National Formulary 'Treatment of Glaucoma' were extracted [7]. For each item listed two data points were extracted; number of prescriptions made in that year and the net ingredient cost. As this study focused on the frequency and cost of prescriptions alone, no further data from PCA reports were required. Using data from the office for national statistics this allowed the calculation of an annual prescription frequency for each item, normalised for population [8]. As precise data were not available for the population of England in 2018 at the time of analysis an estimate of 56 million was made based on the trend from preceding years. Items prescribed less than once per thousand population per year were excluded from analysis (Supplementary table 1). For each item cost at time of prescription was normalised to the year 2015 using the contemporaneous consumer price index [9]. 2015 was not selected as a benchmark for any other reason than it being the year selected by the office for national statistics in their presentation of the data. For each item and each year it was prescribed the molecule, pharmacological group to which it belongs, presence of an item brand and presence of preservatives was noted. This dataset prevented trends in the frequency and cost of prescriptions from being obscured by changes in the BNF listed title of items over the 10-year period.

Analyses were performed using SPSS v.24 (IBM Corporation, New York, USA). The Kendall's tau correlation coefficient was used to analyse the significance of trends over the decade observed as the data were nonparametric and not always monotonic.

\section{Results}

Twenty-three different single and combination agents were used in the treatment of glaucoma and prescribed at a greater frequency than once per thousand population per year from 2009 to 2018. These prescribed items belonged to five pharmacological groups (prostaglandin analogues (PGA), beta blockers (BB), carbonic anhydrase inhibitors

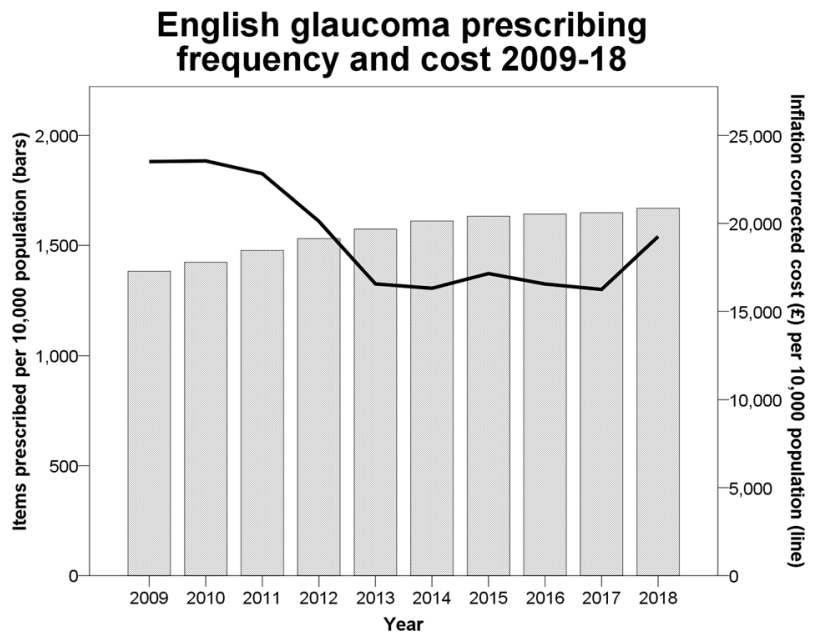

Fig. 1 Total population corrected frequency of glaucoma prescriptions in primary care in England over time (bars) with the associated inflation corrected cost (line)

(CAI), alpha agonists (AA), and anti muscarinics (AM)) with four different combinations $(\mathrm{CAI} / \mathrm{BB}, \mathrm{AA} / \mathrm{BB}, \mathrm{PGA} /$ $\mathrm{BB}$, and $\mathrm{CAI} / \mathrm{AA})$. Four different preservatives were used (benzylkonium chloride, ionic buffered preservative (Sof$\mathrm{Zia}^{\circledast}$ ), stabilised oxychloro complex (Purite ${ }^{\circledast}$ ) and polyquaternium-1).

One thousand six hundred and sixty-one primary care prescriptions were made per 10,000 population at a cost of $£ 114.2$ million in 2018. The annual number of prescriptions normalised for population increased year on year (correlation coefficient $=1.00, P<0.001)$ from 2009 to 2018 (Fig. 1). The proportion of generic items prescribed trended significantly from $11.7 \%$ in 2009 to $55.2 \%$ in 2018 (correlation coefficient $=0.956, P<0.001$ ), whilst their contribution to total glaucoma prescribing spending increased from 4.4 to $37.0 \%$. The proportion of preservative free items prescribed also increased each year (correlation coefficient $=1.00, P<0.001$ ) from 1.7 to $13.9 \%$ between 2009 and 2018 whilst their contribution to total glaucoma prescribing cost increased from 2.8 to $21.0 \%$. Two of the top ten most commonly prescribed items in 2018 were preservative free (Table 1).

Inflation and population corrected costs showed an insignificant decreasing trend (correlation coefficient $=$ $-0.60, P=0.16$ ) over the whole 10 -year period largely due to the substantial drop in 2012 outweighing a smaller rise in 2018 (Fig. 1). These fluctuations mirror the price of latanoprost $0.005 \%$ which made up $28.7 \%$ of all glaucoma prescriptions made in 2018 (Table 1). It came off patent in 2012, leading to a near fivefold decrease in unit price. Between April and June of 2018 the unit cost of latanoprost $0.005 \%$ rose from $£ 2.46$ to a peak of $£ 19.73$ from which it gradually fell through the remainder of 2018 (Fig. 2). 
Table 1 Top ten most commonly prescribed topical glaucoma treatments in English primary care in 2018

\begin{tabular}{llc}
\hline BNF listed name of item & $\begin{array}{l}\text { Number of 2018 } \\
\text { prescriptions }\end{array}$ & $\begin{array}{l}2018 \\
\text { Cost (£) }\end{array}$ \\
\hline Latanoprost_Eye Dps $50 \mathrm{mcg} / \mathrm{ml}$ & $2,164,470$ & 10.98 \\
Lumigan_Eye Dps $100 \mathrm{mcg} / \mathrm{ml}$ & 898,542 & 17.30 \\
Brinzolamide_Eye Dps $10 \mathrm{mg} / \mathrm{ml}$ & 851,952 & 3.08 \\
Ganfort_Eye Dps $300 \mathrm{mcg} / 5 \mathrm{mg}$ & 821,572 & 20.88 \\
Dorzolamide/Timolol_Eye Dps & 461,956 & 2.56 \\
20 mg/5 mg/ml & & \\
Monopost_Eye Dps 50 mcg/ml & 406,233 & 11.37 \\
0.2 mlUd P/F & & 11.01 \\
Travoprost_Eye Dps $40 \mathrm{mcg} / \mathrm{ml}$ & 341,286 & 6.23 \\
Latanoprost/Timolol_Eye Dps & 268,484 & 3.54 \\
50 mcg/5 mg/ml & & 17.52 \\
Brimonidine Tart_Eye Dps 0.2\% & 256,803 & \\
Lumigan_Eye Dps 300 mcg/ml & 244,832 & \\
0.4 ml Ud & & \\
\hline BNF Britih Nationa Formylary & & \\
\hline
\end{tabular}

$B N F$ British National Formulary

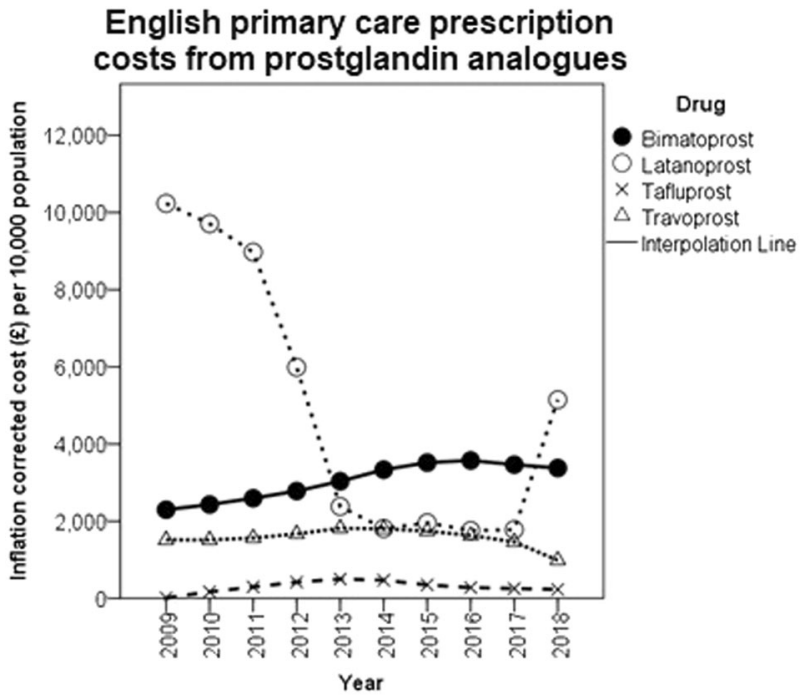

Fig. 2 Total spending on prostaglandin analogue prescriptions by molecule in England

The number of combination items prescribed increased each year (correlation coefficient $=1.00, P<0.001$ ) from 265 to 478 per 10,000 population from 2009 to 2018, with the largest rise seen in PGA/BB combinations (Fig. 3). There were also significant increasing trend in the annual frequency of PGA (correlation coefficient $=0.659, P=$ 0.009 ) and CAI (correlation coefficient $=1.00, P<0.001$ ) whilst BB (correlation coefficient $=-0.99, P<0.001$ ) and AM monotherapy (correlation coefficient $=-0.97, P<$ 0.001 ) became less frequent over the decade studied. There was no significant trend in AA monotherapy prescription frequency (correlation coefficient $=0.00, P=1.00$ ).
Glaucoma prescription frequency by therapeutic class

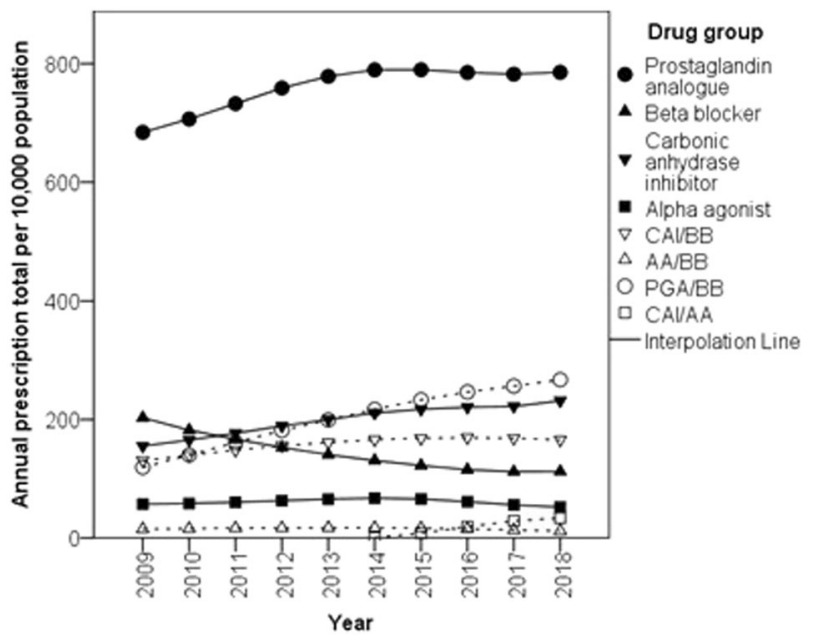

Fig. 3 Annual frequency of glaucoma item prescriptions per 10,000 population categorised by pharmacological group. CAI carbonic anhydrase inhibitor, BB beta blocker, PGA prostaglandin analogue, AA alpha agonist

\section{Discussion}

The total number of glaucoma prescriptions has increased over the past 10 years but the recent rate of increase has slowed. The 2009 NICE glaucoma and OHT guideline was followed by a joint guideline from the colleges of optometrists and ophthalmologists recommending secondary care referral for most individuals with an intraocular pressure higher than $21 \mathrm{mmHg}$ [10]. This represented a large change in the case finding behaviour of optometrists and hugely increased the number of referrals to hospital eye services [11]. Not all of the patients referred under these recommendations would have subsequently required treatment but it is the most likely source of the increase in prescribing seen from 2009. Subsequent slowing of the increase may represent stabilisation of the referral process and equilibrium being reached with people entering and leaving the hospital eye services. It may also represent the increased use of combination agents lowering the absolute number of items prescribed. Although no clear change is yet evident in 2018, this equilibrium is likely to continue to adjust following the 2017 NICE guidelines which advised against referral of ocular hypertension with intraocular pressure under $24 \mathrm{mmHg}$. This amendment should reduce the number of low risk patients referred but may not have a dramatic effect in prescription numbers as many of these patients will not have required treatment under the 2009 NICE guideline. This will be countered by a more balanced rise in referrals due to the increasing age of the UK population and the doubling of glaucoma prevalence seen for each decade of age increase [12]. Other demographics, such 
as socioeconomic status and ethnicity, may also come to have an impact if there are significant shifts [13].

The recommendation from the 2017 NICE guideline to use generic prescriptions as first line treatment reflected a preexisting trend in practice. This is the main explanation for the relative stability of glaucoma prescribing costs up until 2017 despite the increase in prescribing frequency. The clearest deviation from this pattern has been the use of branded bimatoprost although as bimatoprost lost its patent protection in 2017 the importance of this will likely diminish. If bimatoprost items were substituted for generic latanoprost items, inflation corrected costs in 2017 would have been $£ 31.4$ million lower (34.7\% of all 2017 glaucoma prescribing cost). 2017 data are used to illustrate this point as 2018 is much less representative of the 5 year preceding pattern because of the spike in latanoprost cost in June of 2018. Following communication with the UK Department of Health and Social Care the authors understood this spike to be secondary to a temporary shortage of latanoprost $0.005 \%$ causing the price to rise suddenly. At the recommendation of the Pharmaceutical Services Negotiating Committee, primary care was protected from this cost by a concessionary price which was last found to be necessary in February 2019.

As the outcomes of the relevant studies are intraocular pressure differences rather than measures of vision or quality of life, generalisable health economics analyses are not available. However, meta-analysis suggests around a $0.5 \mathrm{mmHg}$ difference between bimatoprost and latanoprost monotherapy [14]. This is roughly a third of both the inter and intra observer variation seen in Goldmann tonometry [15]. A preference for bimatoprost over latanoprost is further limited by the associated increase in orbital fat atrophy and hyperaemia [14, 16, 17].

The revision in the 2017 guideline to recommend preservative free prescriptions for patients with symptomatic ocular surface disease reflects a preexisting increase in their use. This revision also reflects the most recent edition of the European Glaucoma Society guidelines and so we could expect the increasing prescription of preservative free items to apply to Europe more broadly [18]. This trend is costly and the extent to which it is justified cannot be established from these data. The evidence base for preservative free glaucoma drops is largely preclinical and in the few double blinded clinical trials that have taken place ocular surface signs and symptoms have been equivocal [19-22]. However in the context of preexistent dry eye disease, where there are no double blinded clinical trials, what evidence exists does suggest symptomatic improvement from switching to preservative free formulations [22]. This low level evidence forms the basis of the recommendation for a targeted use of preservative free glaucoma medications. In order to more completely appraise current practice, data on the proportion of preservative free prescriptions made in the context of symptomatic ocular surface disease would be necessary. In the absence of this information prescribers should be mindful of patients' ocular surface symptoms and consider trialling alternative agents within the same therapeutic class before preservative free preparations. If all preservative free prescriptions in 2018 were exchanged for a BAK containing preparation of the same drug $£ 13.0$ million (11.4\% of total 2018 spend) would be saved. Whilst a proportion of these preservative free prescription may well be benefiting the recipients, some will have been prescribed with no impact on the ocular surface symptoms caused by the inherent toxicity of topical glaucoma medicines. The equivocal clinical effect of all preservatives in use despite their differing toxicity profiles in preclinical studies is also of importance economically [22]. Both preservative free and BAK free drops are substantially more costly than BAK containing generic equivalents.

The present study is limited by its biased sampling method. A more comprehensive review of English prescribing practice would consider hospital, FP10 and private prescriptions, none of which are publicly available with the same level of detail. Given the frequency of hospital clinic and inpatient visits made by a typical individual with glaucoma, and the low proportion of English glaucoma care provided in the private sector, the present study is still likely to be representative of wider practice. It is also true that the present study was not able to consider the number of treatment doses that each item prescription would offer. 'Quantity' is a measure of this found in PCA reports but as the unit which it represents varies between items, e.g., number of ampoules or millilitres of solution, it could not be applied as a comparator across all prescriptions. Another significant limitation is the inability to associate prescribing practice with indications, such as the mechanism of ocular hypertension, prior treatment inefficacy or the presence of ocular surface disease. With current coding practices in the UK it is hard to see how these data could be made available on a large enough scale as to be informative. However, if trends towards the dominance of electronic health records continue, facilitating data coding at source by clinicians, real world insights into the cost-benefit profile of these agents may become available in the future.

In conclusion both the emphasis on generic PGA as first line treatment and the broadening of preservative free preparation use recommendations in the 2017 NICE glaucoma guideline are being reflected in practice. Due to a temporary eightfold increase in the unit cost of generic latanoprost this preference has been costly in 2018, but 2017 data suggest as much as $£ 31.4$ million could still be saved annually by preferential prescription of generic treatments. The selection of preservative-free glaucoma drops over preserved equivalents cost English primary care services $£ 13.0$ million in 2018. This appears out of proportion with the supporting evidence base, particularly given the continuing growth of 
this expense, and may negate the financial benefits to the NHS of patent expirations.

\section{Summary}

\section{What was known before}

- English primary care glaucoma prescription frequency and cost had been increasing up until 2012.

- The 2017 Glaucoma NICE guideline encouraged generic prescribing and broadened the indication for preservative-free eye drops.

- The evidence base to support preservative free glaucoma drops to manage ocular surface symptoms or switching agents within therapeutic class to further drop intraocular pressure is weak.

\section{What this study adds}

- Glaucoma prescription frequency continues to climb despite increased use of combination agents.

- Although 2018 was an anomaly generally the cost of glaucoma prescribing has decreased over the last 10 years mainly due to generic latanoprost use.

- Half of glaucoma prescribing costs now come from the selection of branded or preservative free agents over their cheapest equivalent in therapeutic class.

Funding Unrelated 2018 travel grant for JH from Bayer TM

\section{Compliance with ethical standards}

Conflict of interest The authors declare that they have no conflict of interest.

Ethical approval All the data presented is in the public domain, no confidential or patient identifiable material was used at any stage of this work. Consequently ethical approval was not sought.

Publisher's note Springer Nature remains neutral with regard to jurisdictional claims in published maps and institutional affiliations.

\section{References}

1. Tham YC, Li X, Wong TY, Quigley HA, Aung T, Cheng CY. Global prevalence of glaucoma and projections of glaucoma burden through 2040: a systematic review and meta-analysis. Ophthalmology. 2014;121:2081-90.

2. Royal National Institute for the Blind., Eye health and sight loss stats and facts, 2018. www.rnib.org.uk/professionals/knowledge-a nd-research-hub/key-informationand-statistics.

3. Connor AJ, Fraser SG. Glaucoma prescribing trends in England 2000 to 2012. Eye. 2014;28:863-9.
4. The King's Fund, Briefing; Deficits in the NHS, 2006. www. kingsfund.org.uk/sites/default/files/field/field_publication_file/ briefing-deficits-in-nhs-kings-fund-jun06.pdf.

5. National Institute for Health and Care Excellence, Glaucoma: diagnosis and management, 2017.

6. NHS Digital, Prescription Costs Analysis, In: Department of Health (ed), 2018. digital.nhs.uk/data-and-information/publica tions/statistical/prescription-cost-analysis.

7. British National Formulary, London: BMJ Group and Pharmaceutical Press, 2018.

8. Office for National Statistics., Population estimates, 2018. www. ons.gov.uk/peoplepopulationandcommunity/populationandmigra tion/populationestimates/timeseries/enpop/pop

9. Office for National Statistics., CPI Index 00: All items $2015=$ $100,2018$.

10. The Colleges of Optometrstis and Ophthalmologists, Guidance on the referral of Glaucoma suspects by community optometrists, 2010.

11. Edgar DF, Romanay T, Lawrenson JG, Myint J. Referral behaviour among optometrists: increase in the Number of Referrals from optometrists Following the Publication of the April 2009 NICE Guidelines for the Diagnosis and Management of COAG and OHT in England and Wales and its Implications. Optom Pract. 2010;11:33-38.

12. Kapetanakis VV, Chan MPY, Foster PJ, Cook DG, Owen CG, Rudnicka AR. Global variations and time trends in the prevalence of primary open angle glaucoma (POAG): a systematic review and meta-analysis. Br J Ophthalmol. 2016;100:86-93.

13. Heng JS, Wormald R, Khaw PT. Geographical variation in glaucoma prescribing trends in Wngland 2008-2012: an observational ecological study. BMJ Open. 2016;6:e010429.

14. Cheng JW, Wei RL. Meta-analysis of 13 randomized controlled trials comparing bimatoprost with latanoprost in patients with elevated intraocular pressure. Clin Therapeutics. 2008;30:622-32.

15. Dielemans I, Vingerling JR, Hofman A, Grobbee DE, de Jong PTVM. Reliability of intraocular pressure measurement with the Goldmann applanation tonometer in epidemiological studies. Graefe's Arch Clin Exp Ophthalmol. 1994;232:141-4.

16. Van Der Valk R, Webers CAB, Schouten JSAG, Zeegers MP, Hendrikse F, Prins MH. Intraocular pressure-lowering effects of all commonly used glaucoma drugs: a meta-analysis of randomized clinical trials. Ophthalmology. 2005;112:1177-85.

17. Aihara M, Shirato S, Sakata R. Incidence of deepening of the upper eyelid sulcus after switching from latanoprost to bimatoprost. Jpn J Ophthalmol. 2011;55:600-4.

18. European Glaucoma Society, Chapter 3.3 Antiglaucoma Drugs, Terminology and Guidelines for Glaucoma: PubliComm, 2014.

19. Shedden A, Adamsons IA, Getson AJ, Laurence JK, Lines CR, Hewitt DJ, et al. Comparison of the efficacy and tolerability of preservative-free and preservative-containing formulations of the dorzolamide/timolol fixed combination (COSOPT ${ }^{\mathrm{TM}}$ ) in patients with elevated intraocular pressure in a randomized clinical trial. Graefe's Arch Clin Exp Ophthalmol. 2010;248:1757-64.

20. Day DG, Walters TR, Schwartz GF, Mundorf TK, Liu C, Schiffman $\mathrm{RM}$, et al. Bimatoprost $0.03 \%$ preservative-free ophthalmic solution versus bimatoprost $0.03 \%$ ophthalmic solution (Lumigan) for glaucoma or ocular hypertension: A 12-week, randomised, doublemasked trial. Br J Ophthalmol. 2013;97:989-93.

21. Goldberg I, Gil Pina R, Lanzagorta-Aresti A, Schiffman RM, Liu C, Bejanian M. Bimatoprost $0.03 \% /$ timolol $0.5 \%$ preservative-free ophthalmic solution versus bimatoprost $0.03 \% /$ timolol $0.5 \%$ ophthalmic solution (Ganfort) for glaucoma or ocular hypertension: A 12-week randomised controlled trial. Br J Ophthalmol. 2014;98:926-31.

22. Steven DW, Alaghband P, Lim KS. Preservatives in glaucoma medication. Br J Ophthalmol. 2018;102:1497-503. 